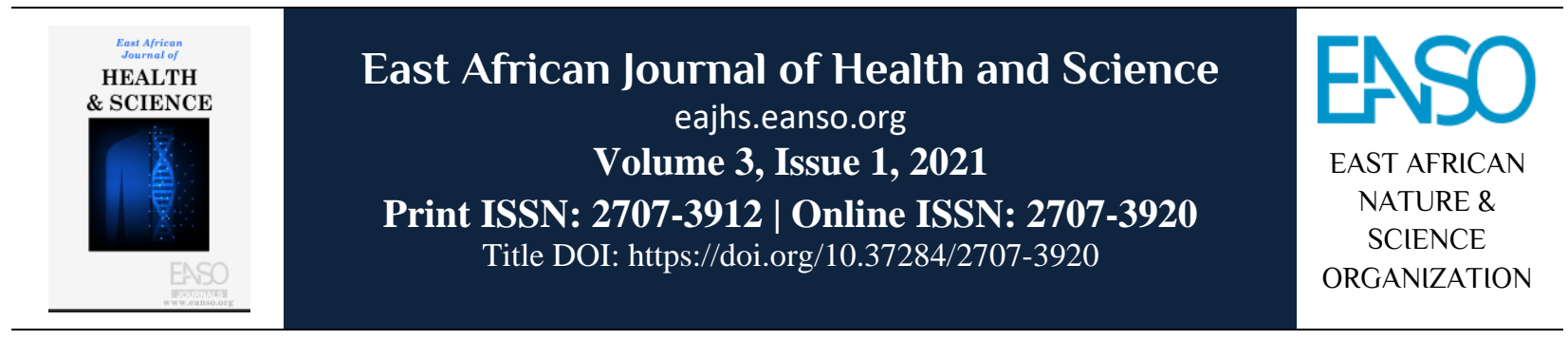

Original Article

\title{
Sexual Practices and HIV Seropositivity Disclosure among Adults in Nairobi, Kenya
}

\author{
Phoebe D. Ndayala, PhD \& Lucy W. Ngige, PhD ${ }^{*, 2}$, Alice Ondigi, PhD $D^{3} \&$ Humphries Evelia 4 \\ ${ }^{1}$ Kenyatta University, P. O. Box 43844 - 00100, Nairobi, Kenya; ORCID: https://orcid.org/0000-0002-2406-8989. \\ ${ }^{2}$ Kenyatta University, P. O. Box 43844 - 00100, Nairobi, Kenya; ORCID: https://orcid.org/0000-0003-1750-1496. \\ ${ }^{3}$ Kenyatta University, P. O. Box 43844 - 00100, Nairobi, Kenya; ORCID: https://orcid.org/0000-0002-2595-5474. \\ ${ }^{4}$ Globus Associates Questream, P. O. Box 745 - 00100, Nairobi, Kenya; ORCID: https://orcid.org/0000-0001-9718-937. \\ *Author for correspondence email: pdndayala@gmail.com.
}

Article DOI: https://doi.org/10.37284/eajhs.3.1.265

\section{Date Published: ABSTRACT}

07 January 2021 The purpose of the study was to assess the relationship between sexual practices and HIV seropositive status disclosure to sexual partners by People Living with

Keywords: Human Immune-Deficiency Virus (PLWHIV) in Nairobi, Kenya. A survey was

HIV and AIDS, HIV Seropositivity, Self-Disclosure; Sexual Practices. conducted among 232 PLWHIV who were registered members of HIV support groups in an informal settlement. Results showed that half $(50.5 \%)$ of the respondents had disclosed their HIV seropositive status to their sexual partners. Chi-square results revealed statistically significant relationships between HIV seropositive disclosure and the following sexual behaviours: condom use in the last sexual encounter $\left(\chi^{2}=12.144 ; \mathrm{df}=1 ; p=0.001\right)$; regular sexual partner $(\chi 2=5.124$; $\mathrm{df}=1 ; p=0.024)$; agreement on HIV testing with sexual partner $\left(\chi^{2}=3.873\right.$; $\mathrm{df}=$ $1 ; p=0.039)$ and knowledge of sexual partners' HIV serostatus $\left(\chi^{2}=6.536\right.$; $\mathrm{df}=$ $1 ; p=0.011)$. The binary logistic regression results established four positive predictors of self-disclosure to sexual partners as regular sexual partner (AOR = 2.506; $p=0.012)$, knowledge of sexual partners' HIV serostatus $(\mathrm{AOR}=3.949 ; p$ $=0.015)$, condom use during the last sexual encounter $(\mathrm{AOR}=3.507 ; p=0.035)$ and agreement on HIV testing with sexual partner (AOR $=2.560 ; p=0.020$ ). However, the desire to conceive (AOR $=3.050 ; p=0.094)$ and the method of testing HIV serostatus ( $\mathrm{AOR}=0.853 ; p=0.530$ ) were not significant predictors of HIV seropositivity disclosure. It was concluded that respondents who knew their partners HIV serostatus and also used a condom during their last sexual encounter were four times more likely to disclose than those who were not aware of partners' serostatus or those who did not use a condom in their last sexual encounter. Furthermore, those who were in regular sexual relationships and also agreed on 
HIV testing with sexual partners were twice more likely to disclose than those in casual sexual relationships. An analysis of sexual practices of PLWHIV can therefore enhance the formulation of targeted strategies aimed at enhancing HIV prevention and reduction of risky sexual behaviour among PLWHIV.

\section{APA CITATION}

Ndayala, P., Ngige, L., Ondigi, A., \& Evelia, H. (2021). Sexual Practices and HIV Seropositivity Disclosure among Adults in Nairobi, Kenya. East African Journal of Health and Science, 3(1), 1-11. https://doi.org/10.37284/eajhs.3.1.265.

\section{CHICAGO CITATION}

Ndayala, Phoebe, Lucy Ngige, Alice Ondigi, and Humphries Evelia. 2021. "Sexual Practices and HIV Seropositivity Disclosure among Adults in Nairobi, Kenya". East African Journal of Health and Science 3 (1), 1-11. https://doi.org/10.37284/eajhs.3.1.265.

\section{HARVARD CITATION}

Ndayala, P., Ngige, L., Ondigi, A., and Evelia, H. (2021) "Sexual Practices and HIV Seropositivity Disclosure among Adults in Nairobi, Kenya", East African Journal of Health and Science, 3(1), pp. 1-11. doi: 10.37284/eajhs.3.1.265.

\section{IEEE CITATION}

P. Ndayala, L. Ngige, A. Ondigi, and H. Evelia, "Sexual Practices and HIV Seropositivity Disclosure among Adults in Nairobi, Kenya”, EAJHS, vol. 3, no. 1, pp. 1-11, Jan. 2021.

\section{MLA CITATION}

Ngige, Lucy, Phoebe Ndayala, Alice Ondigi, and Humphries Evelia. "Sexual Practices and HIV Seropositivity Disclosure among Adults in Nairobi, Kenya". East African Journal of Health and Science, Vol. 3, no. 1, Jan. 2021, pp. 1-11, doi:10.37284/eajhs.3.1.265.

\section{INTRODUCTION}

HIV and AIDS remain one of the serious global public health challenges (U.S. Department of Health \& Human Services, 2019). Southern and Eastern Africa is home to $53 \%$ of People Living with HIV (PLWHIV) globally (UNAIDS, 2019). In 2019, this region accounted for $45 \%$ of new HIV infections marking the region as the most affected by the epidemic globally. Kenya had the thirdlargest joint global HIV epidemic in the world alongside Tanzania (UNAIDS, 2019). According to the National AIDS Control Council (NACC, 2018) estimates, the Kenya national adult HIV prevalence rate was estimated at $4.9 \%$ with higher prevalence rates among women (5.2\%) compared to their male counterparts (4.5\%). According to the report, the total number of people living with HIV in Kenya was estimated at approximately 1.5 million in 2017 (NACC, 2018). Since a large percentage of Kenya's population is affected or infected with HIV, there is need to promote HIV seropositivity disclosure in order for the country to halt the spread of the epidemic and to attain the zero new infections target for epidemic control.
HIV disclosure which is the act of notifying one's Human Immune-Deficiency Virus (HIV) status to someone else is a reasoned process where the costs and benefits to one and others are weighed (Stutterheim et al., 2011). According to Ngula and Miller (2010), the renewed focus on HIV testing and disclosure as a preventive and risk reduction strategy in national HIV programmes has led to its growth in prominence in recent years. Customarily, disclosure is considered as a dilemma; on the one hand, it can stimulate psychological well-being and support, but on the other hand, it can result in rejection, stigmatization and other harmful social interactions (Stutterheim et al., 2011). According to a study carried out in Kenya by Neville and Rubin (2007) among PLWHIV, the decision to notify someone about their seropositive status means evaluating enormous potential for either positive or negative consequences. Disclosure of HIV status is perceived as a way of decreasing risky sexual behaviour, transmission of the virus, reduction in HIV-related stigma and increased access to support and care.

According to Wamoyi et al. (2011), a focus on HIV seropositive disclosure is crucial given that Kenya's HIV epidemic is generalized, driven by sexual 
transmission and the advanced quality of life of PLWHIV on uptake of antiretroviral therapy (ART) stimulates the desire to regain normal reproductive health needs (Sarna et al., 2009). Studies imply that despite knowing their seropositive status, a large proportion of PLWHIV engages in unprotected sex with HIV partners who are negative or partners of unknown status putting them at risk of getting infected (NACC and National AIDS and STI Control Programme (NASCOP), 2007; NASCOP $\& \mathrm{MoH}, 2012)$. This is exacerbated further by evidence from a study on HIV risk reduction behaviours in gay men (Jin et al., 2009) and a national survey by NASCOP \& Ministry of Health $(\mathrm{MoH})(2012)$ which indicated that many PLWHIV engage in risky sexual behaviour which may lead to an escalation in HIV infections. A study among PLWHIV carried out in Mombasa Kenya indicated that $62 \%$ of the respondents reported having unprotected sex with regular sexual partners of HIV-negative or unknown HIV status (NASCOP \& $\mathrm{MoH}, 2012$ ). In addition, the participants who had not disclosed their HIV seropositivity were also more likely to have multiple sexual partners or HIV seronegative partners and also engaged in unprotected sex with non-concordant sexual partners (NASCOP \& MoH, 2012). This calls for a need to design targeted and effective health interventions aimed at equipping PLWHIV with skills to practice safe sex and mitigate risks associated with seropositive status non-disclosure to sexual partners.

According to NACC (2018) report, HIV prevalence in Kenya is declining; however, there is a marked increase in the numbers of PLWHIV experiencing prolonged lives principally due to highly effective treatment regimens and other countrywide interventions initiated to address the HIV scourge. Kenya has also witnessed a high number of discordant and concordant couples calling for a focus on prevention with HIV positives in the fight against new HIV infections. The HIV prevalence in Nairobi County was estimated at $6.1 \%$ which is above the National estimated adult HIV prevalence rate of $4.9 \%$. The report indicated that of the estimated total new infections $(52,800)$ in 2017, Nairobi contributed the highest recording 7,159 new infections (NACC, 2018). Evidence from a population-based HIV prevalence survey carried out in Kenya (Madise et al., 2012) found that the
HIV prevalence was high at $12 \%$ among slum residents compared to 5\% among non-slum urban population and $6 \%$ among rural residents. A study conducted by Dodoo et al. in 2019 reported that residents from informal settlements were less likely to be aware of preventive measures against HIV and AIDS. Therefore, the objective of this study was to determine whether there was a statistically significant relationship between sexual practices and HIV seropositive status disclosure to sexual partners among PLWHIV. The null hypothesis posited that sexual practices among PLWHIV do not predict HIV seropositivity disclosure.

\section{MATERIALS AND METHODS}

A survey was conducted among 232 randomly selected respondents who were HIV seropositive, from a community-based support group for PLWHIV in Nairobi, Kenya. A semi-structured interview guide was used to assess sexual practices and prevalence of HIV seropositive status disclosure to sexual partners among PLWHIV. The independent variables were represented by the knowledge of sexual partners' HIV serostatus, the number of sexual partners and the consistent use of condom during sexual intercourse among the respondents. The dependent variable was HIV seropositive status self-disclosure to sexual partners. Ethical approvals were obtained from the relevant research authorities as well as informed written consent from the respondents prior to the study. Interviews were conducted in order to obtain perspectives on sexual practices and HIV seropositive status disclosure to sexual partners. The Cronbach alpha measure of internal consistency for all the research items ranged from .70 to .82 .

\section{RESULTS}

Results indicate that a total of 232 respondents participated in the study comprising $72.0 \%$ females and $28.0 \%$ males. The mean age of respondents was 39.9 and the median was 40 years. The distribution of marital status showed that $27.6 \%$ were currently living with their sexual partners while $13.4 \%$ were single, $26.3 \%$ separated, $4.3 \%$ divorced and $28.4 \%$ widowed. Approximately one-fifth $(20.3 \%)$ of the respondents were in formal employment while $79.7 \%$ were in the informal sector. In terms of 
education, over two thirds (69.8\%) had attained a primary level of education, $23.3 \%$ had completed secondary education, while $6.9 \%$ had achieved tertiary education.

\section{Knowledge of HIV Prevention and Sexual Practices among PLWHIV}

The distribution of knowledge of HIV prevention methods and the sexual practices among the respondents is presented in Table 1 .

Table 1: Knowledge of HIV Prevention and Sexual Practices among PLWHIV

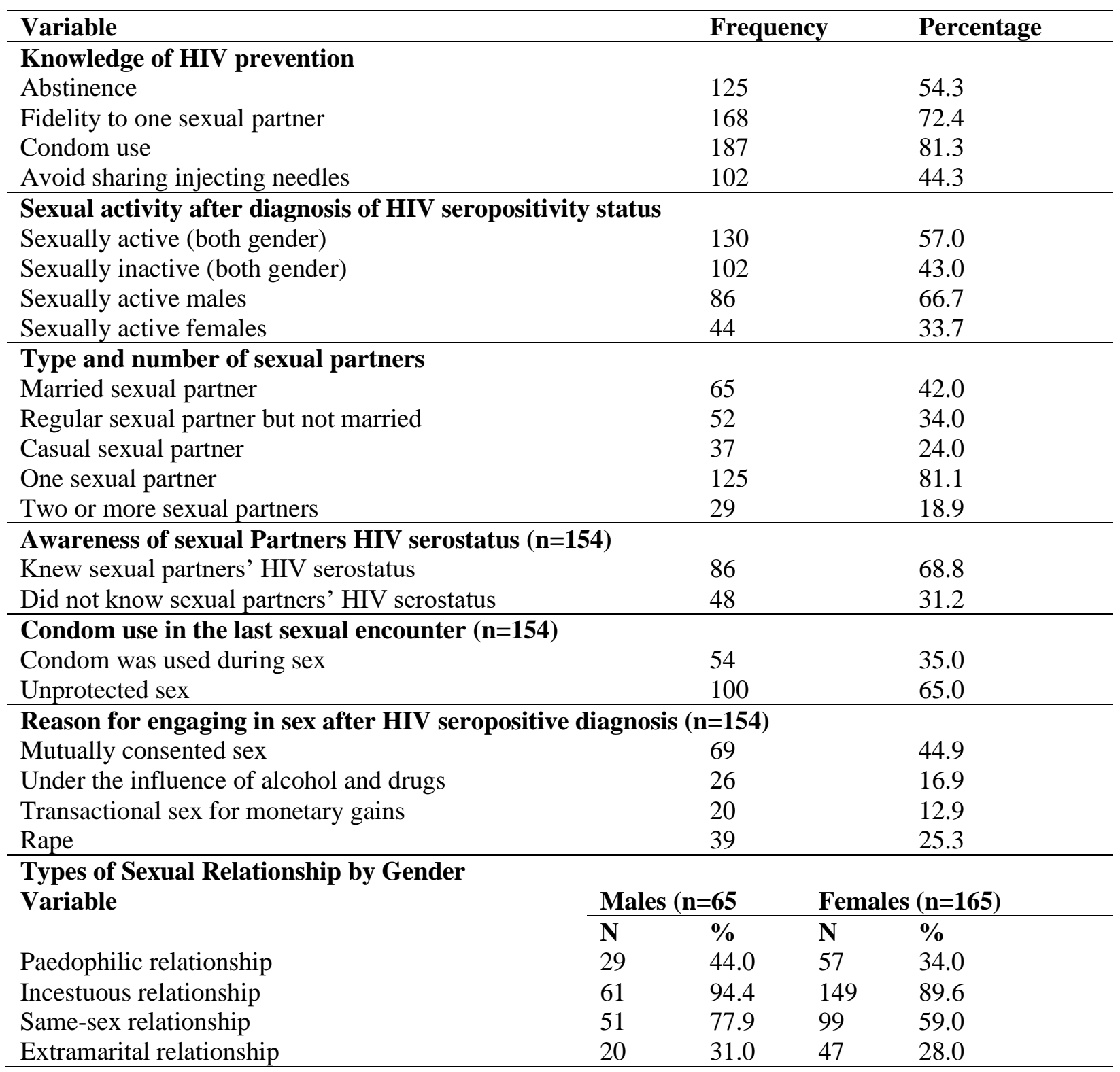

*Multiple responses included 


\section{Knowledge of HIV Re-Infection Prevention Methods}

Results presented in Table 1 showed that knowledge of methods of preventing re-infection among PLWHIV included abstinence (54.3\%), fidelity to one sexual partner $(72.4 \%)$, condom use $(81.3 \%)$, and to avoid sharing contaminated injecting needles (44.3\%) (Table 1). Knowledge of HIV re-infection prevention strategies among respondents was fairly high and therefore, it was hoped that they were likely to increase their uptake of safer sex practices.

\section{Sexual Activity among PLWHIV and HIV Self- disclosure}

Respondents were considered sexually active when they reported any form of sexual intercourse in the last six months prior to the study. Over half $(57 \%)$ reported that they were sexually active while $43.0 \%$ indicated that they were sexually inactive. When asked if they had ever engaged in sexual intercourse with anyone since they discovered their HIV seropositive diagnosis, $74.1 \%$ reported that they had while $25.9 \%$ had not. Results revealed that most respondents $(68.8 \%)$ were aware of their current sexual partners' serostatus, while $31.2 \%$ were not aware (Table 1). Disaggregation by gender indicated that $66.3 \%$ of the males engaged in sexual intercourse even after knowing their HIV seropositive status compared to $33.7 \%$ of their female counterparts.

\section{Type of Current Sexual Relationship for PLWHIV}

Of the 154 respondents who recorded having sexual partners at the time of the study, $42 \%$ were married, $24 \%$ had an occasional sexual partner, $34 \%$ were in a regular sexual relationship but not married. The majority $(86 \%)$ were in a regular sexual relationship while slightly over one fifth $(24 \%)$ engaged in casual sexual relationships (Table 1).

\section{Number of Sexual Partners for PLWHIV}

The number of sexual partners an individual has is an important indicator of risky sexual behaviour. Out of 154 respondents who reported having sexual partners at the time of the study, $81.1 \%$ reported having one sexual partner only, while $18.9 \%$ reported having two or more sexual partners. This indicates that although the majority recorded monogamous sexual relationships at the time of the study, cases of multiple sexual partners were observed.

\section{Reasons for First Sexual Encounter after HIV Diagnosis by PLWHIV}

After knowing their HIV seropositive status diagnosis, majority of the respondents (44.9\%) engaged in mutually consented sexual encounters, $25.3 \%$ reported that they were raped, $16.9 \%$ reported engaging in sex under the influence of alcohol and drugs, and $12.9 \%$ had transactional sex for monetary gains (Table 1). This implied that in the case of rape or when one was under the influence of alcohol, disclosure of serostatus and use of protection was least likely to occur which may have placed sexual partners at risk of infection and PLWHIV at risk of re-infection.

\section{Condom Use During the Last Sexual Encounter}

Condom use during the last sexual encounter was low as slightly over a third (35\%) of the respondents who were sexually active at the time of the study stated that they had used a condom during their last sexual encounter. Results indicated that approximately two- thirds $(65.0 \%)$ engaged in unprotected sex even after knowing their HIV seropositive status (Table 1). Majority of the participants expressed unwillingness to use condoms during sexual encounters for varied reasons captured in the verbatim reports such as reduced sexual satisfaction and the desire to conceive.

"Why eat a wrapped sweet when your partner has "mdudu" like you. You just enjoy sex for the short time you are with her." (FGD-3).

"I cannot use condoms because I want to have a baby. I do not want to die and have nobody to carry on my family lineage." (FGD-4).

\section{Motivation for Undergoing HIV Testing}

Focus group discussion reports revealed that the motivation for undergoing HIV testing was not an act of voluntary initiative, but rather necessitated by either health-related infection management such as 
persistent cough, weight loss and fever. Others took the HIV test as a requirement for other health processes such as the prevention of mother-to-child transmission (PMTCT) during routine maternal health care clinics, while for others it was a prerequisite for a job interview or as a pre-marital counselling requirement. This means that a large proportion of the PLWHIV discovered their serostatus through a Provider Initiated Testing and Counselling (PITC) initiative rather than a Voluntary Counselling and Testing (VCT) effort. In addition, the main reason for HIV seropositive status disclosure was to access health care services and or for social and economic support from significant others.

\section{Prevalence of HIV Testing and Discussion on HIV Testing with Sexual Partner}

Voluntary Counselling and Testing (VCT) is an important component in HIV prevention efforts. Majority of the respondents (93.1\%) had discussed HIV testing with their sexual partners while $6.9 \%$ had never discussed HIV testing with their sexual partners. Respondents were asked to state whether their current sexual partner had ever undergone an HIV test. Over two thirds $(71.7 \%)$ reported that their current sexual partner had been tested for HIV serostatus, while $28.3 \%$ had never tested for HIV.

\section{Types of Sexual Relationships among PLWHIV}

The results revealed that the respondents practised a variety of sexual relationships. The sexual relationship reported by male respondents included paedophilic (44\%), incestuous $(94.4 \%)$, same-sex (77.9\%) and extramarital relationship (31\%). The sexual relationship reported by female respondents included paedophilic (34\%) incestuous (89.6\%), same-sex (59\%) and extramarital relationships (28\%). The findings implied that the majority of the male and female respondents engaged in either incestuous relationships or same-sex partnerships, about one-third engaged in paedophilic relationships and slightly over one-quarter reported extramarital relationship. During focus group discussions, the respondents concurred that these sexual relationships were considered as cultural taboos in the community.

\section{Prevalence of HIV Seropositivity Disclosure to Sexual Partners}

The distribution of HIV seropositive status disclosure among sexual partners is presented in Table 2.

Table 2: HIV seropositive status disclosure among sexual partners

\begin{tabular}{llc}
\hline & Frequency & Percentage \\
\hline HIV seropositive status disclosure to sexual partners (n-232) & & \\
Prevalence of HIV seropositive status disclosure & 117 & 50.5 \\
HIV seropositive status non-disclosure & 115 & 49.5 \\
\hline Knowledge of current sexual partners' HIV serostatus (n=146) & & \\
HIV Seropositive (Concordant) & 86 & 55.8 \\
HIV Sero-negative (Discordant) & 20 & 13.0 \\
Unknown HIV serostatus & 40 & 31.2 \\
\hline
\end{tabular}

Results indicated that approximately half $(50.5 \%)$ of the respondents had ever disclosed their seropositive status to sexual partners while $49.5 \%$ had not disclosed. However, only $29.9 \%$ of those who reported being sexually active at the time of the study stated that they consistently disclosed their seropositive status to all sexual partners. Knowledge of sexual partners' HIV serostatus was deemed to enhance HIV seropositive status disclosure. Of the respondents who reported knowing the serostatus of their sexual partners, $55.8 \%$ stated that their sexual partners were HIV seropositive while $13.0 \%$ were HIV seronegative and $31.2 \%$ did not know their sexual partners' HIV serostatus. This implies that the majority of sexual partners were HIV seropositive and concordant with the respondents' HIV serostatus. Results from the focus group discussion indicated that HIV testing among sexual partners was common among 
the respondents in this area as reported by some female respondents:

"We are encouraged to advise our partners to go for HIV testing in order to know their status and make decisions on how to live" (FGD-1).

"I informed him that I was HIV positive and advised him that we should go for testing at the
VCT centre together. He agreed and we went together and he also tested positive" (FGD-2).

\section{Hypothesis Test Results}

The null hypothesis stated that there was no statistically significant relationship between sexual practices and HIV serostatus disclosure among PLWHIIV. The chi-square test results are presented in Table 3.

Table 3: Chi-square results for sexual practices and HIV serostatus self-disclosure

\begin{tabular}{|l|l|l|l|}
\hline Variables & Chi-square & $(\mathbf{d f})$ & Sig. $(\mathbf{p}$-value) \\
\hline Being sexually active & $\chi^{2}=0.003$ & $\mathrm{df}=1$ & $p=0.956$ \\
\hline Knowledge of sexual partners' HIV serostatus & $\chi^{2}=6.536$ & $\mathrm{df}=1$ & $p=0.011^{*}$ \\
\hline HIV concordant sexual partners & $\chi^{2}=6.536$ & $\mathrm{df}=1$ & $p=0.011^{*}$ \\
\hline Desire to conceive after HIV seropositive diagnosis & $\chi^{2}=15.564$ & $\mathrm{df}=1$ & $p=0.004^{*}$ \\
\hline Having multiple sexual partners & $\chi^{2}=0.012$ & $\mathrm{df}=1$ & $p=0.914$ \\
\hline Regular sexual relationship & $\chi^{2}=5.124$ & $\mathrm{df}=1$ & $p=0.024^{*}$ \\
\hline Condom use during last sexual encounter & $\chi^{2}=12.144$ & $\mathrm{df}=1$ & $p=0.001^{*}$ \\
\hline Mutual agreement on HIV testing with sexual partner & $\chi^{2}=3.873$ & $\mathrm{df}=1$ & $p=0.049^{*}$ \\
\hline PITC-initiated HIV testing & $\chi^{2}=4.272$ & $\mathrm{df}=1$ & $p=0.049^{*}$ \\
\hline Incestuous relationships & $\chi^{2}=15.797$ & $\mathrm{df}=1$ & $p=0.001^{*}$ \\
\hline Same-sex partnerships & $\chi^{2}=14.167$ & $\mathrm{df}=1$ & $p=0.001^{*}$ \\
\hline
\end{tabular}

*Significant at $p \leq 0.05$

The results revealed that there were statistically significant relationships between HIV seropositive disclosure and knowledge of current sexual partners' HIV serostatus $\left(\chi^{2}=6.536\right.$; df $=1 ; p=$ $0.011)$, sexual partners who are HIV concordant $\left(\chi^{2}\right.$ $=6.536 ; \mathrm{df}=1 ; p=0.011)$; Desire to conceive after HIV seropositive diagnosis $\left(\chi^{2}=15.654 ; \mathrm{df}=1 ; p\right.$ $=0.004)$, having a regular sexual partner $\left(\chi^{2}=\right.$ 5.124; $\mathrm{df}=1 ; p=0.024)$; condom use in the last sexual encounter $\left(\chi^{2}=12.144\right.$; df $\left.=1 ; p=0.001\right)$, mutual agreement on HIV testing with sexual partner $\left(\chi^{2}=3.873 ; \mathrm{df}=1 ; p=0.049\right)$ and PITC initiated HIV testing $\left(\chi^{2}=4.272 ; \mathrm{df}=1 ; p=0.049\right)$. The sexual relationships that were significantly related to self-disclosure of HIV serostatus were incestuous relationships $\left(\chi^{2}=15.797, \mathrm{df}=1, p=\right.$ $0.001)$ and same-sex partnerships $\left(\chi^{2}=14.167\right.$, $\mathrm{df}=1, \quad p=0.001)$. However, being currently sexually active $\left(\chi^{2}=0.003 ; \mathrm{df}=1 ; p=0.956\right)$ and having multiple sexual partners $\left(\chi^{2}=0.012, \mathrm{df}=1\right.$, $p=0.914$ ) were not significantly related to HIV seropositivity disclosure to sexual partners.
The study established that respondents who were aware that their sexual partners were HIV concordant were more likely to disclose their HIV serostatus compared to those who were in HIV discordant relationships. Respondents who engaged in consensual sexual encounters after HIV seropositive diagnosis were more likely to disclose their seropositive status than those whose sexual encounter non-consensual as a result of rape. Results support the view that respondents were more likely to disclose their serostatus to regular sexual partners or spouses than to casual and occasional sexual partners. Results established that the respondents who reported using a condom during their last sexual encounter were more likely to disclose than those who did not use condoms during sex. These results imply that being in a concordant relationship, having a regular sexual partner, engaging in a consensual sexual relationship, use condoms during sexual encounters, incestuous relationships and same-sex partnerships had a significant relationship with HIV seropositivity status disclosure. These findings were subjected to further analysis using binary 
logistic regression to establish whether sexual practices among PLWHIV predicted HIV seropositivity disclosure. The prediction results are presented in Table 4.

Table 4: Predictors of HIV seropositive status disclosure among sexual partners

\begin{tabular}{llll}
\hline Variables & (AOR) & p-Value & 95\% of CI \\
\hline Regular sexual relationship & 2.506 & $0.012^{*}$ & $1.223-5.133$ \\
Desire to conceive after HIV status diagnosis & 3.050 & 0.094 & $0.826-11.261$ \\
PITC-initiated HIV testing & 0.853 & 0.530 & $0.519-1.1402$ \\
Mutual agreement on HIV testing with a sexual partner & 2.560 & $0.020^{*}$ & $1.981-6.680$ \\
Knowledge of current partners' HIV serostatus & 3.949 & $0.015^{*}$ & $1.309-11.910$ \\
Condom use during the last sexual encounter & 3.507 & $0.035^{*}$ & $1.092-11.263$ \\
Incestuous relationship & 2.746 & 0.191 & $0.604-12.481$ \\
Same-sex partnerships & 0.225 & $0.001^{*}$ & $0.103-0.493$ \\
\hline
\end{tabular}

*Significant predictors at $p \leq .05$

The regression results established that there were four significant positive predictors of HIV seropositive status disclosure to sexual partners comprised of having a regular sexual partner (AOR $=2.703 ; p=0.046$ ), mutual agreement on HIV testing with sexual partner $(\mathrm{AOR}=2.560 ; p=$ 0.020), knowledge of sexual partners' HIV seropositive status (AOR $=3.949 ; p=0.015$ ) and condom use in the last sexual encounter $(\mathrm{AOR}=$ $3.507 ; p=0.035$ ). The results revealed that samesex partnership was a negative predictor of selfdisclosure of HIV seropositivity status to sexual partners $(\mathrm{AOR}=0.225 ; p=0.001)$. However, the desire to conceive after HIV status diagnosis (AOR $=3.050 ; p=0.094$ ) and incestuous relationships were positive predictors $(\mathrm{AOR}>1.000)$, but they were not statistically significant. PITC-initiated HIV testing (AOR $=0.853 ; P=0.530)$ was a negative predictor $(A O R<1.000)$ but it was not statistically significant. These results implied that those who knew their sexual partners' HIV serostatus and used a condom during their last sexual encounter were four times more likely to disclose than those who were not aware of their sexual partners' HIV serostatus and those who did not use a condom during their last sexual encounter. The results further showed that those in regular sexual relationships were two and a half times more likely to disclose their HIV seropositive status than those in casual sexual relationships. This implies that respondents who engaged in same-sex partnerships were less likely to disclose their HIV seropositivity status compared to their counterparts engaged in opposite-sex relationships.

\section{DISCUSSION}

This study has established that approximately half of the respondents had disclosed their HIV seropositivity status to their sexual partners. This finding concurs with studies conducted in Mombasa, Kenya by Sarna et al. (2009) and in Addis Ababa, Ethiopia by Dessalegn et al. (2019). The higher disclosure rates may be attributed to the support, care and training received from the HIV community-based support groups. The study also established that majority of the respondents were currently sexually active with one regular sexual partner. This finding does not concur with other studies by NACC and NASCOP (2007), Otieno (2008) and $\mathrm{Vu}$ et al. (2012) which reported that majority of PLWHIV engaged in risky sexual behaviour with multiple sexual partners. However, it was observed that about one-fifth of the respondents engaged in unprotected sexual encounters with multiple sexual partners even after knowing their HIV seropositive status. This finding is consistent with results of a study by Sarna et al. (2009) among PLWHIV in Mombasa which showed that the majority of PLWHIV continued to engage in unprotected sex even after HIV seropositive diagnosis.

Even though rates of HIV self-disclosure to current sexual partners was moderate, majority of PLWHIV applied selective disclosure to concordant and regular sexual partners and not to casual, occasional or transactional sexual partners. This finding is supported by research findings by Larkins et al. (2005) which indicated that PLWHIV 
in sexual relationships were more likely to disclose their HIV serostatus to regular sexual partners compared to casual sexual partners. The uptake of condom use during the last sexual encounter by PLWHIV was found to promote HIV seropositivity disclosure. This finding is supported by results of a study conducted by Seid et al. (2012) which showed that the exchange of information about one's HIV serostatus with a sexual partner is associated with safer sexual practices. However, these results were inconsistent with research conducted in London by Dave et al. (2006) which indicated that there was no association between HIV seropositive status disclosure and the uptake of safer sex practices among people living with HIV.

Due to the dilemma of informing a sexual partner of HIV seropositive diagnosis poses concerns about negative consequences such as stigma and discrimination against PLWHIV, disclosure was more probable among couples who were concordant than those who were discordant. Results established that most of the PLWHIV engaged in sexual encounters that were consensual after knowing their HIV seropositive diagnosis. However, in some instances where sexual encounters were non-consensual or when one was intoxicated, the decision to disclose the HIV serostatus and the use of condoms was less likely, thereby putting their sexual partners at risk of HIV infection and also the possibility or re-infecting themselves with a different strain of the HIV. Discussion of HIV testing with sexual partners promoted seropositive status disclosure as this was assumed to enhance openness in communication on sexual issues. These findings were supported by other researchers (Seid et al., 2012; Deribe et al., 2010; Kadowa \& Nuwaha, 2009) who reported that the exchange of information about one's HIV status and communication about safe sex with a sexual partner were significantly associated with HIV serostatus disclosure and safer sexual practices.

The motivation for HIV serostatus disclosure found in this study was consistent with findings of a study in the Netherlands by Stutterheim et al. (2011) which showed that disclosure was motivated by the need to attain emotional release, to access financial and social support, or as a perceived duty to inform the other sexual partners. Results of a study carried out in Uganda by Kadowa and Nuwaha (2009) support the current finding that Provider Initiated Testing and Counselling (PITC) was significantly related to HIV serostatus disclosure to sexual partners. This implies that there are benefits accruing from PITC because it is often accompanied by professional counselling which may help improve the self-efficacy of PLWHIV to disclose HIV serostatus to sexual partners. This study established that same-sex partnerships were negative predictors of disclosure of HIV seropositivity status to sexual partners. This implies that same-sex relationships were more likely to inhibit HIV disclosure and escalate HIV infection among sexual partners. This finding concurs with Micheni et al., (2017) who reported that the prevalence of HIV in homosexual men is three times that of the overall populace in Kenya. Furthermore, same-sex relationships are illegal in Kenya (Government of Kenya, 2012).

\section{CONCLUSION}

This study has shown that HIV seropositive status disclosure was higher among respondents who were concordant, in regular sexual relationships, those who used condoms in last sexual encounter and those who knew their sexual partners' HIV serostatus. It was concluded that respondents who knew their sexual partners' HIV serostatus and those who used a condom during their last sexual encounter were four times more likely to disclose than those who were not aware of partners' HIV serostatus and those who did not use a condom in their last sexual encounter. Furthermore, those who were in regular sexual relationships and also discussed HIV testing with sexual partners were more likely to disclose than those in casual or samesex relationships. An analysis of sexual practices of PLWHIV can therefore enhance the formulation of targeted strategies aimed at enhancing the prevention of risky sexual behaviours and escalation of HIV infections. It is therefore recommended that continuous and consistent counselling is vital to ensure informed decisions on safer sex practices and HIV prevention among PLWHIV. 


\section{ACKNOWLEDGEMENT}

We wish to appreciate the respondents who participated in this study.

\section{REFERENCES}

Dessalegn, N. G., Hailemichael, R. G., ShewaAmare, A., Sawleshwarkar, S., Lodebo, B., Amberbir, A., \& Hillman, R. J. (2019). HIV Disclosure: HIV-positive status disclosure to sexual partners among individuals receiving HIV care in Addis Ababa, Ethiopia. PloS one, 14(2), e0211967.

Dave, S. S., Stephenson, J., Mercey, D. E., Panahmand, N., \& Jungmann, E. (2006). Sexual behaviour, condom use, and disclosure of HIV status in HIV infected heterosexual individuals attending an inner London HIV clinic. Sexually Transmitted Infections, 82(2), 117-119.

Deribe, K., Woldemichael, K., Njau, B. J., Yakob, B., Biadgilign, S., \& Amberbir, A. (2010). Gender differences regarding barriers and motivators of HIV status disclosure among HIV-positive service users. SAHARA-J: Journal of Social Aspects of HIV/AIDS, 7(1), 30-39.

Dodoo, F. N. A., Zulu, E. M., \& Ezeh, A. C. (2007). Urban-rural differences in the socioeconomic deprivation-Sexual behavior link in Kenya. Social Science \& Medicine, 64(5), 1019-1031.

Government of Kenya. (2012). Penal code of 1930, Laws of Kenya, CAP. 63 (Revised, 2012).

Jin, F., Crawford, J., Prestage, G. P., Zablotska, I., Imrie, J., Kippax, S. C. \& Grulich, A. E. (2009). HIV risk reduction behaviours in gay men: unprotected anal intercourse, risk reduction behaviours, and subsequent HIV infection in a cohort of homosexual men. AIDS, 23(2), 243252.

Kadowa, I., \& Nuwaha, F. (2009). Factors influencing disclosure of HIV positive status in Mityana district of Uganda. African Health Sciences, 9(1), 26-33.

Larkins, S., Reback, C. J., Shoptaw, S., \& Veniegas, R. (2005). Methamphetamine- dependent gay men's disclosure of their HIV status to sexual partners. AIDS Care, 17(4), 521532.

Madise, N. J., Ziraba, A. K., Inungu, J., Khamadi, S. A., Ezeh, A., Zulu, E. M. \& Mwau, M. (2012). Are slum dwellers at heightened risk of HIV infection than other urban residents? Evidence from population-based HIV prevalence surveys in Kenya. Health \& Place, 18(5), 1144-1152.

Micheni, M., Kombo, B.K., Secor, A., Simoni, J.M., Operio, D., Van der Elst, E.M., Mugo, P., Kanungi, J., Sanders, E.J. \& Graham, S.M. (2017). Health provider views on improving antiretroviral therapy adherence among men who have sex with men in coastal Kenya AIDS Patient Care and STDs, Vol. 31, Number 3, 2017 Available at DOI: 10.1089/apc.2016.0213.

National AIDS Control Council (NACC). (2018). HIV estimates report for Kenya. Retrieved from https://nacc.or.ke/wpcontent/uploads/2018/11/HIV-estimates-reportKenya-20182.pdf

National AIDS Control Council (NACC0 \& National AIDS and STD Control Programme (NASCOP). (2007). National HIV prevalence in Kenya._Nairobi: GOK.

National AIDS and STI Control Programme (NASCOP) and MoH, Kenya (2012). Kenya AIDS Indicator Survey (KAIS): Nairobi, Kenya: National AIDS and STI Control Programme (NASCOP) and MoH, Kenya.

Neville, M. A. \& Rubin, D. L. (2007). Factors leading to self-disclosure of a positive HIV diagnosis in Nairobi, Kenya: people living with HIV/AIDS in the Sub-Sahara. Qualitative Health Research, 17(5), 586-598.

Ngula, K. W., \& Miller, A. N. (2010). Selfdisclosure of HIV seropositivity in Kenya by HIV-positive Kamba men and their families. Southern Communication Journal, 75(4), 328-348.

Otieno, B.A, (2008). Effects of Antiretroviral Therapy on sexual behavior among people living with HIV and AIDS in Kibera slums in 
Nairobi, Kenya. 2008; Unpublished MPH thesis: Kenyatta University.

Sarna, A., Chersich, M., Okal, J., Luchters, S. M., Mandaliya, K. N., Rutenberg, N., \& Temmerman, M. (2009). Changes in sexual risk taking with antiretroviral treatment: influence of context and gender norms in Mombasa, Kenya. Culture, Health \& Sexuality, 11(8), 783-797.

Seid, M., Wasie, B., \& Admassu, M. (2012). Disclosure of HIV positive result to a sexual partner among adult clinical service users in Kemissie district, northeast Ethiopia. African Journal of Reproductive Health, 16 (1). 97-104.

Stutterheim, S. E., Shiripinda, I., Bos, A. E., Pryor, J. B., de Bruin, M., Nellen, J. F. \& Schaalma, H. P. (2011). HIV status disclosure among HIVpositive African and Afro-Caribbean people in the Netherlands. AIDS Care, 23(2), 195-205.

UNAIDS (2018). Global HIV \& AIDS Statistics 2019 fact sheet. Retrieved from https://www.unaids.org.

UNAIDS (2019). AIDS information. Retrieved from https:///aidsinfo.unaids.org.

U.S. Department of Health \& Human Services. (2019). Global HIV/AIDS. Retrieved from https://www.hiv.gov/federal-response/pepfarglobal-aids/global-hiv-aids.

Vu, L., Andrinopoulos, K., Mathews, C., Chopra, M., Kendall, C., \& Eisele, T. P. (2012). Disclosure of HIV status to sex partners among HIV-infected men and women in Cape Town, South Africa. AIDS and Behavior, 16(1), 132138.

Wamoyi, J., Mbonye, M., Seeley, J., Birungi, J., \& Jaffar, S. (2011). Changes in sexual desires and behaviour of people living with HIV after initiation of ART: Implications for HIV Prevention and health promotion. BMC Public Health, 11, 633. 